\title{
Demo: A system for image acquisition and processing operating in the visible and the IR bands
}

\author{
J.A. Leñero-Bardallo \\ Inst. Microelectrónica de \\ Sevilla (IMSE-CNM), \\ CSIC-Univ. Sevilla (Spain) \\ Tel. +34954466666 \\ juanle@imse- \\ cnm.csic.es
}

\author{
J. Bernabéu-Wittel \\ Hospital Virgen de Rocío, \\ Sevilla (Spain) \\ Tel. +34661980850 \\ jbernabeuw@gmail.com
}

\author{
J. Ceballos-Cáceres \\ Inst. Microelectrónica de \\ Sevilla (IMSE-CNM), \\ CSIC-Univ. Sevilla (Spain) \\ Tel. +34954466666 \\ joaquin@imse- \\ cnm.csic.es
}

\author{
Á. Rodríguez-Vázquez \\ Inst. Microelectrónica de \\ Sevilla (IMSE-CNM), \\ CSIC-Univ. Sevilla (Spain) \\ Tel. +34 954466666 \\ angel@imse- \\ cnm.csic.es
}

\begin{abstract}
This demo displays an autonomous image acquisition and processing system that operates simultaneously with two image sensors either in the visible and the Long Wave Infrared Band (LWIR), inside the Infrared (IR) band. The entire system is controlled a Raspberry Pi board that allows to easily program image processing algorithms to process the images acquired with each sensor. It is a competitive alternative to conventional commercial closed systems with infrared cameras. The proposed imaging system can be easily adapted to different operation scenarios by adding new peripherals, sensors and full custom image processing algorithms.
\end{abstract}

\section{CCS Concepts}

- Hardware $\rightarrow$ Communication Hardware, interfaces and storage $\rightarrow$ Sensors and actuators.

\section{Keywords}

Multi-spectral imaging; IR camera; Image Processing; Camera interface; Raspberry Pi.

\section{INTRODUCTION}

There are many application scenarios in which multispectral analysis combining images of the visual scene in the visible and the Infrared (IR) band is demanded. Infrared cameras can provide thermal information of the visual scene and can operate during the night without any illumination. Thus, there are a clear alternative to conventional CMOS cameras when their performance is compromised by the illumination conditions or in application scenarios where local temperature variations need to be measured precisely. For instance, LIDAR systems can benefit of IR cameras to detect pedestrians during the night [1]. Surveillance systems can employ IR cameras to detect intruders without illumination [2]. There is also a growing interest the analysis of biomedical images in different radiation bands [3].

Permission to make digital or hard copies of part or all of this work for personal or classroom use is granted without fee provided that copies are not made or distributed for profit or commercial advantage and that copies bear this notice and the full citation on the first page. Copyrights for thirdparty components of this work must be honored. For all other uses, contact the Owner/Author.

ICDSC '19, September 9-11, 2019, Trento, Italy @ 2019 Copyright is held by the owner/author(s). ACM ISBN 978-1-4503-7189-6/19/09.

https://doi.org/10.1145/3349801.3357127
Regarding IR image sensors, during the last decade, they have reduced significantly their cost. We can find affordable low and medium resolution IR sensors [4] with a price below \$300. There are also different providers that already commercialize systems that mount simultaneously CMOS and IR cameras. However, their cost is still quite high and these solutions are not clearly open to embed and program multispectral image processing algorithms or add new sensors. Furthermore, such systems do not offer the possibility of acquiring and displaying simultaneously images in different spectral bands. To be competitive in the implementation of systems with smart cameras, they must allow several functions. Just to name but a few, they should have the possibility of processing images, store the processes images on a memory, transmit them wirelessly or activate actuators. In that sense, the Raspberry Pi board [5] is a perfect platform to control several cameras and process their outputs simultaneously.

In this demo, we will showcase a portable and autonomous system with two image sensors. The first one is a CMOS camera and the second one is a thermographic camera from the LEPTON manufacturer. Both sensors are controlled by a Raspberry Pi board. The images acquired by them are displayed, processed and stored simultaneously by two independent $\mathrm{C}++$ interfaces that are open and can be customized to implement any image processing algorithm in the visible or either in the LWIR band. In that sense, the image processing functions available in the OpenCV repository [6] can be directly incorporated to the system.

During the demo, the visitor will have the possibility of holding the system, that operates autonomously with a battery, and acquiring, and displaying simultaneously video in the visible and LWIR band. The spectators will also have the opportunity of performing a realtime sensor interface configuration. Some basic image processing algorithms implemented in the LWIR band will be shown and explained to them. Detailed information about the system design will be provided. Interfaces' source code will be available for download.

Overall, we present a powerful, affordable, portable, autonomous and configurable system for multispectral image acquisition and processing. It operates in the visible and LWIR band. It will be explained how to download the source code of the sensor interfaces and how to incorporate image processing algorithms to process their outputs. The proposed multi-camera system is a perfect tool to deploy more complex vision systems that require vision in the infrared band and custom image processing algorithms. 


\section{VISITOR'S EXPERIENCE}

In Figure 1, we show in detail the dual camera system final implementation and the custom programmable interfaces available. The visitor will be able to hold and use the system to record video and images in the visible and the IR band simultaneously. Examples of processing algorithms already implemented to detect temperature gradients will be shown. Furthermore, the spectator will have access to links with repositories where the system software can be downloaded. Detailed information about the hardware implementation and the peripherals connectivity will be provided to the attendants.

\section{CONCLUSIONS}

A custom and configurable autonomous multi-spectral imaging system based on a Raspberry Pi board has been showcased. The system is intended to implement real-time image processing algorithms with images taken in the visible and the IR band. It offers the possibility of being expanded by adding new peripherals and sensors through the Raspberry Pi available ports. Thus, it can be easily adapted to specific application scenarios in different fields where IR vision is required.

\section{ACKNOWLEDGMENTS}

This work was supported by Spanish Government MINECO and European Region Development Fund, (ERDF/FEDER) through

(a)

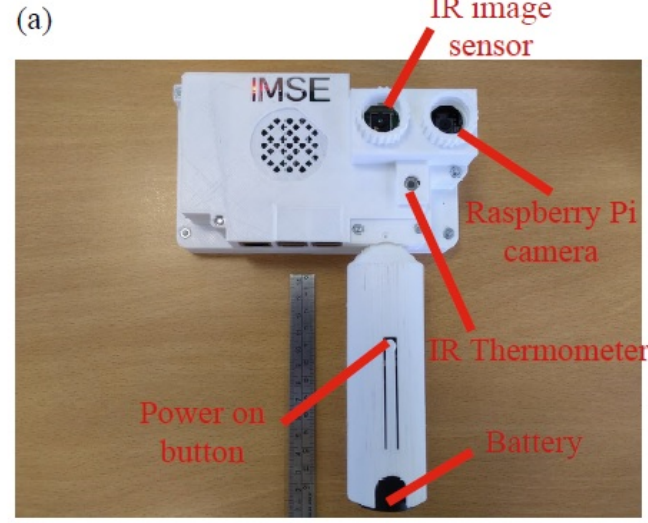

(c)

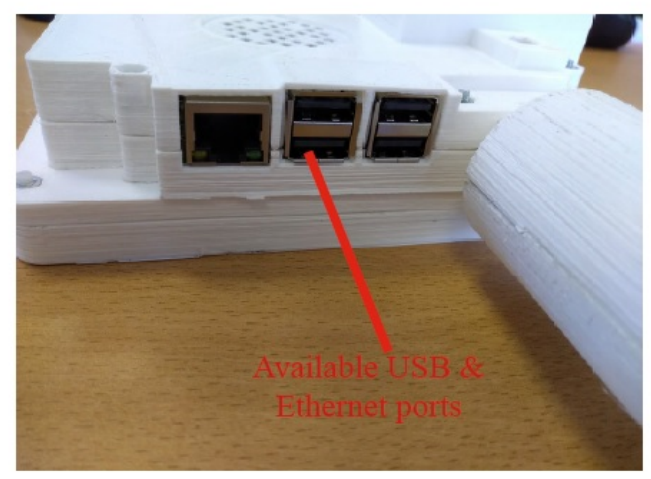

Project RTI2018-097088-B-C31, by Ayudas a Proyectos de I+D+I Programa Operativo FEDER US-1264940, by ONR grant ONR NICOP N00014-19-1-2156 and by EU H2020 MSCA ACHIEVEITN, Grant No 765866.

\section{REFERENCES}

[1] Rajeev Thakur. Scanning LIDAR in Advanced Driver Assistance Systems and Beyond: Building a road map for next-generation LIDAR technology, IEEE Consumer Electronics Magazine, 5(3), 48-54, (2016).

[2] S. J. Krotosky and M. M. Trivedi. Person Surveillance Using Visual and Infrared Imagery. IEEE Transactions on Circuits and Systems for Video Technology, 18(8), 1096 -1105, (2008).

[3] Saxena AK, Willital GH. Infrared thermography: Experience from a decade of pediatric imaging. Eur J Pediatr., 167:757764. (2008).

[4] "FLIR LEPTON sensor interface repository" 2019 https://github.com/groupgets/LeptonModule.

[5] "Raspberry Pi 3 Model B." 2019 https://www.raspberrypi.org/products/raspberry-pi-3-modelb/.

[6] “OpenCV repository” 2019 https://opencv.org/

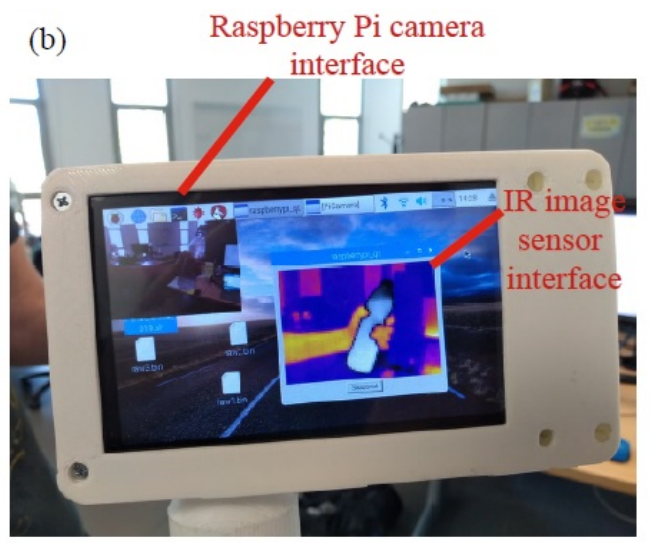

(d)

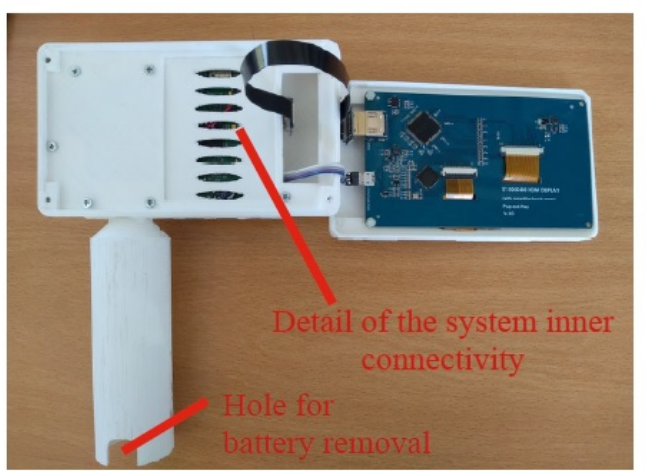

Figure 1. Details of the multi-camera autonomous system that will be exposed and tested during the live demo. (a) Front view. The available sensors and peripherals are indicated. (a) Back view. Detail of the two camera interfaces operating in parallel. (c) Available ports for system connectivity and expansion. (d) Details of the hardware connectivity. 